\title{
Machine learning model-based two-dimensional matrix computation model for human motion and dance recovery
}

\author{
Yi Zhang ${ }^{1} \cdot$ Mengni Zhang $^{1}$
}

Received: 10 February 2020 / Accepted: 6 August 2020 / Published online: 12 September 2020

(c) The Author(s) 2020

\begin{abstract}
Many regions of human movement capturing are commonly used. Still, it includes a complicated capturing method, and the obtained information contains missing information invariably due to the human's body or clothing structure. Recovery of motion that aims to recover from degraded observation and the underlying complete sequence of motion is still a difficult task, because the nonlinear structure and the filming property is integrated into the movements. Machine learning model based two-dimensional matrix computation (MM-TDMC) approach demonstrates promising performance in short-term motion recovery problems. However, the theoretical guarantee for the recovery of nonlinear movement information lacks in the two-dimensional matrix computation model developed for linear information. To overcome this drawback, this study proposes MM-TDMC for human motion and dance recovery. The advantages of the machine learning-based Two-dimensional matrix computation model for human motion and dance recovery shows extensive experimental results and comparisons with auto-conditioned recurrent neural network, multimodal corpus, low-rank matrix completion, and kinect sensors methods.
\end{abstract}

Keywords Computation model $\cdot$ Machine learning $\cdot$ Human motion $\cdot$ Two-dimensional matrix $\cdot$ Neural

\section{Introduction}

Interactive dance has drawn growing attention on the new form of performing arts for choreographers, composers, visual artists, computers, and engineers. This concern has its roots not only in their liberty to communicate with and regulate audio and visual feedback for choreographers and dancers but also in their difficulties of creating feedback motors capable of responding quickly to the dancer's motion $[1,2]$. This interest is built mainly on the free responses to the choreographer and dancers, not on the challenges facing composers and visual performers to develop feedback engines that respond quickly to the dancer movement $[3$, 4]. However, computer scientists find it more difficult to establish robust signal processing and model recognition algorithms based on movement data that dancers use to

Yi Zhang

zhangyi6188@163.com

Mengni Zhang

duyu5643@163.com

1 College of Music, Hubei University of Science and Technology, Xianning 450046, China communicate with feedback engines to regulate audio and visual feedback [5].

For sensing and analyzing human motion, interactive dance represents distinctive difficulties. The exactness of movement analysis, like the use of costume markers, the presence of various dancers, and complicated dance movement, can be determined by many factors in dance performance. Appropriate sensing methods and interactive signals must be chosen, and robust movement analysis algorithms must be developed to allow interactive dance $[6,7]$.

One of the most efficient methods of digitizing human motions and applications in computer graphics is the motion capture system $[8,9]$. However, the system only gives lowlevel data. Motion graphs can generate more variable movements using signal processing methods or with space-times requirements $[10,11]$.

The research is an effort to create a secure algorithm to evaluate the movements of dancers from comprehensive monitoring and group dynamic analysis for a lengthy period to the recognition of dynamic and static physiological postures at several moment and space levels. In the movement analysis studies, several modalities of motion sensing were used, including a motion capture scheme based on markers, pressurized ground. To increase the analytical engine 
recognition range, the pressurized floor and the markerbased motion sensing system are combined.

The significant contributions of this paper are:

- To recover the human motion problems using machine learning model based two-dimensional matrix computation (MM-TDMC) approach with better accuracy.

- To design a computation model for human motion and dance recovery.

- To validate the experimental results of the machine learning model based two-dimensional matrix computation (MM-TDMC) approach in comparison with other methods.

A wide range of applications has some distinct methods for representing human movements and recognition. Analysis of human motion is a more general word, and implementation determines the amount and length of movement of the body components. Human-computer communication usually includes only hand gestures, whereas dance consists of all components of the body, complicated activities, or sports. The conceptual categorization of the human movement into gestures, behavior, tasks, relationships, and group activities is based on complexity. The methodologies of representation and recognition are chosen from the video survey and the initiation of the human body. Comprehensive representational methods are 2-D cinematic or stick figure, 3-D film or form model, and model image. The human initialization using the kinematic technique is described by characteristics such as the number of joints, liberty of the individual, limb length, etc. However, human image is shown in the image model itself, and characteristics such as shape or region are extracted and stored. In addition, the recognition and complexity of the movement can be chosen. Simple activities are using single-layered methods in a sequential and space-time manner. In this manuscript, section "Literature survey" discusses the literature review, and section "Machine learning model based two-dimensional matrix computation (MM-TDMC)" explains the importance of the two-dimensional matrix computation model and description of motion and dance recovery. Section "Experimental results and discussion" briefs about the numerical analysis, and section "Conclusion" concludes the research with future extension.

\section{Literature survey}

As per the survey, in Ref. [12], the authors introduced a method in real-time for the synthesis of highly complex human movements using a new training system, called the auto-conditioned recurrent neural network (acRNN). This method can synthesize highly complex arbitrary movements, including locomotion and dance or martial arts. The acRNN can do this by accommodating automatic noise accumulation during training explicitly. Their work is the first to be able to produce more than 18,000 constant frames of new and complex human motion in different styles (300 s). The result is the main problem was that the technique was not very different from a conventional LSTM.

In Refs. [13], the authors introduced a new multimodal corpus (MMC) for research on real-time, realistic human interactions in the online, virtual environments, among other areas. The various corpus scenarios concentrate on an Internet application of the dance school, in which students can learn choreographies from the teacher's manual at a virtual dance studio, where avatars can be obtained locally by any 3D capture technology. As the dance troops focus on this scenario, it consists of students/teachers' dance choreographies, which are captured simultaneously by different media techniques from two places, such as synchronized audio systems, different cameras, inertial measuring devices, and depth sensors. This MMC technique has low efficiency and dance retrieval system precision.

In Ref. [14], the researchers presented the low-rank matrix completion (LRMC) for the collection of human movement information (MOCAP) that records joint motions of a human body. However, because of capture system constraints, the raw data collected invariably contain missing data [15]. All current techniques use the convex nuclear standards to overcome the resulting issue of ranking minimization known as NP-hard as a ranking replacing the convexity of the objective function. This paper which has high error recovery and human intuition agreement [16].

In Refs. [17, 18], the authors used various kinect sensors (KS) to present a technique of body movement analyses in dance. To solve occlusion and self-occlusion problem monitoring to improve the robustness of skeletal tracking, fusion is the approach proposed. The fused skeleton information is subdivided into five different body parts that are then converted to enable invariant recognition of posture. Each part is produced by performing $K$-means on a wide range of unlabeled positions. Hidden conditional random fields (HCRF) classifiers are used to recognize movement patterns and are combined into body posturing sequences [19].

To overcome the above problems, the machine learning model based two-dimensional matrix computation (MMTDMC) approach is used for recovering the human motion and dance recovery. The advantages of the machine learning-based two-dimensional matrix computation model for human motion and dance recovery shows extensive experimental results and comparisons with a recurrent neural network (acRNN), MMC, LRMC, and KS methods. 


\section{Machine learning model based two-dimensional matrix computation (MM-TDMC)}

In this area, the study discusses the essential technological component that is the two-dimensional matrix computation model, a description of motion and dance recovery.

\section{Issue detailing}

In human development, human development is diagram-oneplotted, and every edge is registered in a human skeleton as the places of every joint, regardless of whether the areas of certain joints may be missing, as the halfway development of the body or clothing methods.

The on-screen character shows in Fig. 1. Our responsibility is to fill in absent places and get a complete development course of action.

Here, we use $I \in S^{3}$ to indicate the directions of a joint in the posture space:

We use $e=\left[\begin{array}{c}I_{1} \\ I_{2} \\ \cdot \\ \cdot \\ \cdot \\ I_{e}\end{array}\right] \in S^{3 e}$

Indicate a casing that contains $d$ joints in a human skeleton. We use $N=\left[I_{1}, I_{2}, \ldots I_{N}\right] \in S^{3 e \times N}$ to signify a movement grouping with absent joints, where $N$ is the $N$ and $I_{j}$ poles, which implies the $j$ th line of the group of motion. The lack of joints is set to 0 in $N$. At that point movement retrieval is changed over to a network finish issue of outcome the basic complete lattice of $N$. Our detailing is to utilize the proposed MM-TDMC to take care of the problem and the structure appears in Fig. 1. before presenting the MMTDMC, the researchers have to exhibit the two-dimensional matrix computation model, which is the solution of demonstrating the Riemannian complex formation of human movement.

\section{Riemannian exponential part}

Figure 2 separation of the geodetic and the Euclidean joint on a circle. These two wrist joints originate separately in the ball sequence from the first and last edge

The analyst can measure the similarity between unit quaternions using the Riemannian distance method. A unit quaternion cannot be visualized by the three-dimensional sphere in a four-dimensional space. Riemannian used in two normalized positions for measuring distance. The human skeleton at the origin shown in Fig. 2 shows the correct elbow joint. The preceding is the Riemannian separation, the last being the Euclidean separation.

The researchers standardize the circle by applying a single unit length for easy calculation and statement. They can usually use the standardized position $I^{n}$ because of the geometric pitch of each joint. In addition, the place $I^{n}$ is defined as standardized, as shown in Eq. (2):

$I^{n}=\frac{I-I_{q}}{\left\|I-I_{q}\right\|_{2}}$.

The Riemannian separation is the most appropriate measure of partition between two points on a Riemannian measurement, where the parent joint is a complete position. Where the Riemannian separation is the most appropriate. The Riemannian separation between $x$ and $y$ is defined as being used for $\left(y \in S^{3}\right)$ and $\left(z \in S^{3}\right)$ the two directions on a 2-dimensional circle:

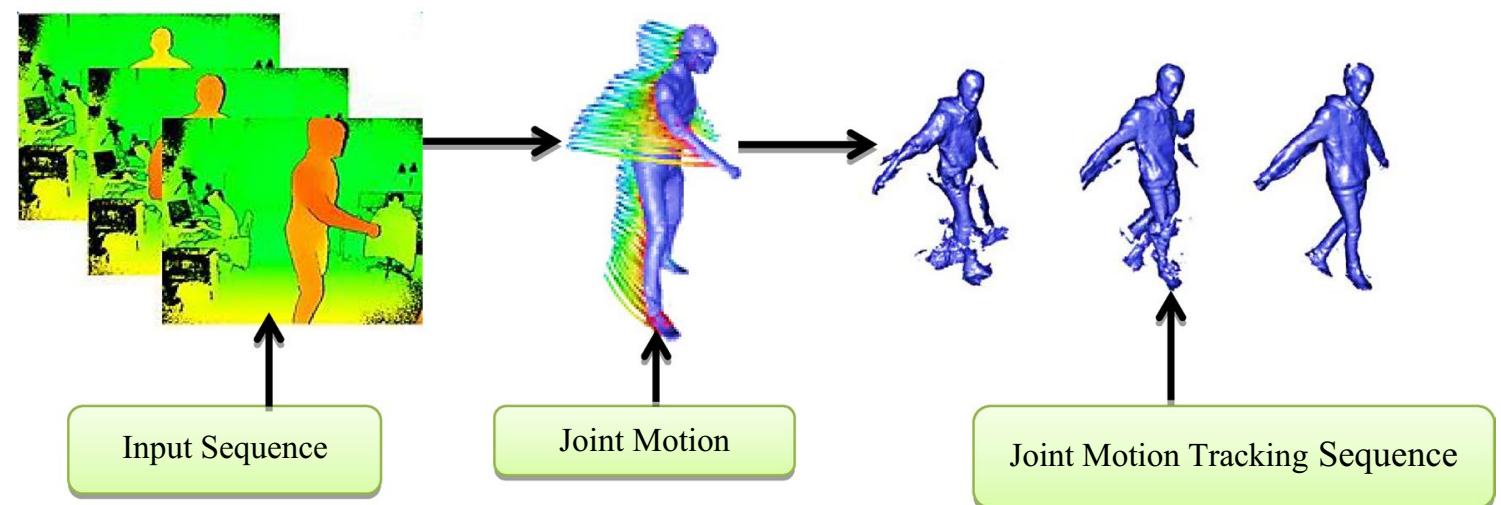

Fig. 1 Dance motion with the halfway development of the body or clothing [20] 


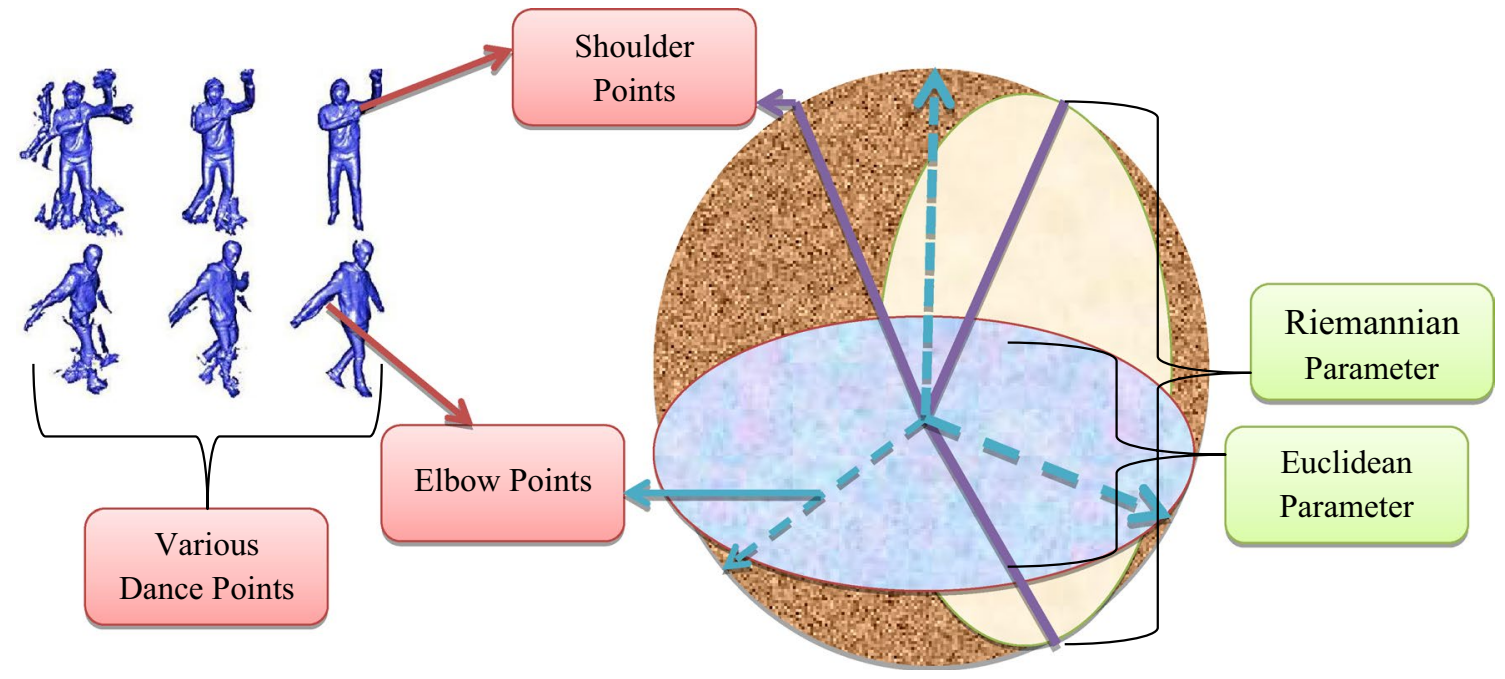

Fig. 2 Riemannian exponential part of dance analysis

$e_{f}(y, z)=\arccos \left(y^{R} z\right)$

From Eq. (3), The normal converse cosine function is $[-1,1][0, \beta]$, where over: $[-1,1]$. The Riemannian exponential bit is defined as being pursued by Riemannian separation:

$g_{\sigma}(y, z)=f^{\frac{-e_{f}(y, z)}{2 \sigma^{2}}}$.

From Eq. (4) Let $\sigma$ as width variable. Considering the Riemannian separation represents the exact calculation of the several movements, the Riemannian exponential piece licenses us to install $\left(S_{n}\right)$ in a top element propagate Kernel Hilbert Space, which increases the precision. For movement catch information, we use $\varphi: e \rightarrow \varphi(e)$ to mean the specific chart characterized by the bit capacity $l_{A}\left(e_{a}, e_{b}\right)$. The inward result of two casings $e_{a}$ an $e_{b}$ is described as in Eq. (5):

$\left\langle\phi\left(e_{a}\right), \phi\left(e_{b}\right)\right\rangle=l_{A}\left(e_{a}, l_{b}\right)$

$\sum_{i=1}^{e} \sum_{n=1}^{X} \alpha_{i n}^{2} l_{\sigma n}\left(I_{a i}^{n}, I_{b i}^{n}\right)$

where $d$ is the quantity of the considerable number of joints in a human skeleton, $I_{a i}^{m}$ and $I_{b i}^{m}$ are individually standardized places of $I_{b i}$ and $I_{a i}, I_{b i}$ and $I_{a i}$ are separately the $i$ th joint in $e_{a}$ and $e_{b}, X$ is the quantity of the width parameters $\sigma m$ which compare to various Riemannian exponential portions $l_{\sigma n}(\cdot, \cdot), \alpha_{i n}^{2}$ is the heaviness of $l_{\sigma n}\left(I_{b i}^{m}, I_{a i}^{m}\right)$ and every one of the loads are sorted in the weight lattice $B$, as shown in Eq. (8):
$B=\left[\begin{array}{ccc}\alpha_{11} & \cdots & \alpha_{1 X} \\ \vdots & \ddots & \vdots \\ \alpha_{e 1} & \cdots & \alpha_{e X}\end{array}\right]_{e X X}$

The part $l_{B}\left(e_{a}, e_{b}\right)$ is intended for different portion understanding, and learning an ideal $B$ is to set human movement into a Hilbert capacity, which is as near its component space as could be expected under the circumstances.

\section{Various types of MM-TDMC}

Straightforwardly inserting movement information into the Hilbert space with one part can not guarantee the estimated level to the component space. Hence, the researchers can utilize various portion learning procedure to get familiar with the element space of human movement. The objective is to look for a lot of loads to join numerous parts (with various width parameters) together, and the at extended last consolidated portion prompts the most shortened position of the movement grid in the Hilbert space. When the learning is done, it implies that the component space is found, and retrieval execution will never again depend on the preparation set, as shown in the matrix (7):

We use $C=\left[e_{1}, e_{2}, \ldots, e_{N E}\right]=\left[\begin{array}{c}C_{1} \\ C_{2} \\ \cdot \\ \cdot \\ \cdot \\ C_{c}\end{array}\right] \in S^{3 e \times N_{E}}$ 
to mean a preparation set made out of a huge number of shapes which originate from different sorts of movement groupings, where $M_{\mathrm{E}}$ is the number of edges in $C, C_{i} \in S^{3 \times M_{E}}$ comparison to all the $i$ th joints in $C$. We use $\varphi(C)$ to indicate the certain mapping of the preparation set $C$ in the Hilbert space. The objective of the various piece learning is to limit the position of $\varphi(C)$, so the target capacity of various portion learning is characterized as

$\min _{B} \operatorname{rank}(\phi(C))$

$\operatorname{diag}\left(B B^{R}\right)=1$,

where rank $(\varphi(C))$ registers the position of $\varphi(C)$ and the requirement is to ensure the last part is a curved blend of various Riemannian exponential pieces. Since Eq. (8) is an l0-standard coordinated rank-minimization issue which is NP-hard, the $l 1$-standard and the atomic standard are frequently utilized as surrogates of the $l 0$-standard and network rank individuals in most lattice execution issues. In any case, the problem is that the understood chart $\varphi(C)$ makes the $l 1$-standard or atomic standard minimization issue immovable, since we cannot legitimately do any task on $\varphi(C)$. Usually, one cannot utilize any typical methodology, for example, solitary worth shrinkage operative to take care of the rank-minimization issue. This way, the study suggests using Schatten $p$-standard $\|.\|_{R_{q}}$ rather than a standard to roughly the position of a grid, as shown in Eq. (9):

$\|Y\|_{R_{q}}=\left(\sum_{j=1}^{\min \{n, m\}} \xi_{j}^{q}\right)^{\frac{1}{q}}=\left(\operatorname{Rt}\left(Y^{R} Y\right)^{\frac{q}{2}}\right)^{\frac{1}{q}}$,

where $q>0, Y \in S^{n \times m}, \xi_{j}$ is the $i$ th single estimation of $Y$. At the point when $q$ is more like 0 , the Schatten $p$-standard $\|.\|_{R_{q}}$ is nearer to the original position of $Y$. At that point, we can loosen up a rank $(\varphi(C))$, as shown in Eq. (10):

$$
\begin{aligned}
\operatorname{rank}(\phi(E)) & \left.\approx\left(\operatorname{Rt}\left(\left((\phi(E))^{R} \phi(E)\right)^{\frac{q}{2}}\right)\right)^{\frac{1}{q}}\right) \\
& =\left(\operatorname{Rt}\left((L(C))^{\frac{q}{2}}\right)\right)^{\frac{1}{q}},
\end{aligned}
$$

where $L(C)$ is the part network of $C$ characterized on $l_{B}\left(e_{a} \cdot e_{b}\right)$. The $\frac{1}{q}$ intensity of $\mathrm{Rt}(\cdot)$ has no impact on the minimization of the position, so for simple calculation, the analysts discard it and rephrase questions (8) as:

$$
\begin{aligned}
& \min _{B} \operatorname{Rt}\left((L(C))^{\frac{q}{2}}\right) \\
& \operatorname{diag}\left(B B^{R}\right)=1 .
\end{aligned}
$$

The target capacity contains a correspondence limitation, so usually, they can utilize the (ALM) augment Lagrange multiplier to take care of the issue. The comparing expanded Lagrange capacity is

$$
\begin{aligned}
Q(B, C, \mu, v)= & \operatorname{Rt}\left((L(C))^{\frac{q}{2}}\right)+\left\langle\mu, \operatorname{diag}\left(B B^{R}\right)-1\right\rangle \\
& +\frac{v}{4}\left\|\operatorname{diag}\left(B B^{R}\right)-1\right\|_{2}^{2} .
\end{aligned}
$$

Here, the analysts solve the optimization problem using the Powell/Hestenes (PH) algorithm. In the PH calculation, minimizing $Q$ with fixed $\mu$ and $\nu$ is the most crucial step:

$\min _{B} Q(B, C, \mu, \nu)$.

Having a considerable $\mu$ and $\nu$, the development issue (12) is simple and arched $\operatorname{around} \operatorname{diag}\left(B B^{R}\right)=1$, which can be effectively unraveled by various inclination based development calculations, for example, the quickened proximal angle technique (APG). The subsidiary of $Q(B, C, \mu, \nu)$ concerning $B$ is shown in Eq. (14):

$$
\begin{aligned}
\frac{\partial Q(B, C, \mu, v)}{\partial B}= & \frac{\partial \operatorname{Rt}\left((L(C))^{\frac{q}{2}}\right)}{\partial B}+\mu^{R} B \\
& +v\left(\operatorname{diag}\left(B B^{R}\right)-1\right)^{R} B
\end{aligned}
$$

As indicated by the chain guideline of framework determination, the term $\frac{\partial R t\left((L(C))^{\frac{q}{2}}\right)}{\partial B}$ can be inferred as in Eq. (15):

$$
\begin{aligned}
& \frac{\partial R t\left((L(C))^{\frac{q}{2}}\right)}{\partial \alpha_{i n}}=\frac{\partial e(H)}{\partial \alpha_{i n}}=\sum_{L}^{M} \sum_{K}^{M} \frac{\partial e(H)}{\partial H_{l k}} \frac{\partial H_{l k}}{\partial \alpha_{i n}} \\
& R t\left(\left(\frac{\partial e(H)}{\partial H}\right)^{R} \frac{\partial H}{\partial \alpha_{i n}}\right),
\end{aligned}
$$

where $\alpha_{i n}$ is the component in the $i$ th push and $n$th segment of $B, H_{l v}$ indicates the element in the $l$ th drive and the $l$ th section of $H, e(H)=R t\left(H^{\frac{q}{2}}\right), H=L(C)$. At that point in Eq. (16):

$\frac{\partial e(H)}{\partial H}=\frac{q}{2} H^{\frac{q-2}{2}}=\frac{q}{2}(L(C))^{\frac{q-2}{2}}$

also

$\frac{\partial H}{\partial \alpha_{i n}}=2 \alpha_{i n} L_{\sigma n}\left(C_{i}\right)$

From Eq. (17), where $L_{\sigma n}\left(C_{i}\right)$ is the piece framework of the $i$ th joint characterized on the part $l_{\sigma n}(\cdot, \cdot)$. When executing the learning procedure, let $q=1 / 16$ for the Schatten $\mathrm{p}$-standard to make an adequately precise estimate of rank (C). As indicated by APG, the analysts give Algorithm 1 to take care of the issue of Eq. (13). At that point, they use a PH calculation to illuminate the entire numerous portion learning issue (11). Since PH calculation is anything but difficult 
to execute, the analysts do not demonstrate the calculation subtleties here yet adjacent it in the strengthening data.

\section{Machine learning model-based two-dimensional matrix computation (MM-TDMC)}

While acquiring the educated portion $l_{b}(\cdot, \cdot)$, which means movement catch, and it has been implanted into its element position. With the educated part, the researchers can actualize the disorganized low-position grid consummation. Accepting that $Y=\left[y_{1}, y_{2}, \ldots, y_{N}\right] \in S^{3 e \times N}$ is the basic complete movement framework of $N$, let $\varphi: Y \rightarrow \varphi(Y)$ to indicate the correct learning mapping characterized on the piece work $l_{b}(\cdot, \cdot)$ in condition (5) and $B$ is acquired by different portion learning. At that point, the kernelized low-position network consummation handles the issue as:
Movements of people constitute a kind of unique grouping of learning data and are the most important to examine. It must meet a few cinematic constraints, or it will be an unnatural recovered motion, which will efficiently be made aware of people. The last streamlining model is shown in Eq. (19):

$$
\begin{aligned}
& \min _{Y} \operatorname{rank}(\phi(Y))+\frac{\beta}{2}\|Y P\|_{e}^{2} \\
& U \circ Y=N \\
& g(Y)=0,
\end{aligned}
$$

where $\beta$ is the tuning parameter, $\frac{\beta}{2}\|Y P\|_{E}^{2}$ is the smoothness imperative term, $P$ is characterized as Eq. (20):

Algorithm 1: Optimization of machine learning parameters:- $\min _{B} Q(B, C, \mu, v)$

Input :

$C, \mu, v, e,\left\{\sigma_{n}\right\}_{n=1}^{X}$, Initialize learning coefficients $\mathrm{B}^{(0)}$ and $\mathrm{L}^{(0)}$ with $\left[\begin{array}{c}1^{R} / \sqrt{e} \\ \vdots \\ 1^{R} / \sqrt{e}\end{array}\right]$, and let

$\theta^{(0)}=1, j=0$

1:repeat

$2: W^{j}=\left(1-\theta^{(j)}\right) B^{(j)}+\theta^{(j)} L^{(j)}$

$3: L^{(j+1)}=L^{(j)}-\frac{1}{e X \theta^{(j)}} \frac{\partial Q\left(W^{(j)}, C, \mu, v\right)}{\partial W^{(j)}}$

$4: B^{(j+1)}=\left(1-\theta^{(j)}\right) B^{(j)}+\theta^{(j)} L^{(j+1)}$

$5: \theta^{(j+1)}=\frac{2}{j+1}$

6: $j=j+1$

$7:$ until convergence or reach the maximum iteration times.

Output: $B^{(j)}$

the piece work $l_{b}(\cdot, \cdot)$ in condition (4) and $\mathrm{B}$ is acquired by different portion learning. At that point, the kernelized low-position network consummation handles the issue as

$\min _{Y} \operatorname{rank}(\phi(Y))$

$U \circ Y=N$,

where $\circ$ is the Hadamard item, $U \in S^{3 e \times N}, U_{j i}=1$ if $N_{j i}$ is watched, $U_{j i}=0$ if $N_{j i}$ is missing. The MM-TDMC-based motion recovery approaches are the most recuperation ranking results. Regular MM-TDMC looks for the negligible $y$ position, but $Y$ may not be of small value given the unorganized structure in motion data. Therefore, the analysts restrict the area of the component (minimize rank $(\alpha(Y))$, which in theory is more and more sensible.

$$
\left[\begin{array}{ccccccc}
-1 & 1 & & & & & \\
1 & -2 & 1 & & & & \\
& \ddots & \ddots & \ddots & & & \\
& & & & 1 & -2 & 1 \\
& & & & & 1 & -1
\end{array}\right]_{M \times M}
$$

The capacity $g(Y)$ is to figure the length of the bone mistake of each joint in $Y$. The length of each bone portion in a human skeleton is decided, so the requirement $g$ $(Y)=0$ can implement the recouped movement to fulfill the bone-length imperative. $g(Y)$ is straightforward, however, 
entangled to portray, so they give the outflow of $g(Y)$ just as its subordinate invaluable data of this study to expand the clarity of this paper.

Not at all, like the target capacity of different portion learning, here the researchers use $\|\varphi(Y)\|_{R 1}(q=1)$ to inexact the position of $\varphi(Y)$, which is curved and has been confirmed to be compelling in the analyses. The condition (19) can be revamped, as shown in Eq. (21):

$$
\begin{aligned}
& \min _{Y} \operatorname{Rt}\left((L(Y))^{\frac{1}{2}}\right)+\frac{\beta}{2}\|Y P\|_{E}^{2} \\
& U \circ Y=N \\
& g(Y)=0 .
\end{aligned}
$$

The target capacity carries two uniformity imperatives, here the researchers can solve the problem using augmented Lagrange multiplier (ALM). The comparing enlarged Lagrange capacity is:

$$
\begin{aligned}
V\left(Y, Z, \delta, \lambda_{1}, \lambda_{2}\right)= & R t\left((L(Y))^{\frac{1}{2}}\right)+\frac{\beta}{2}\|Y P\|_{E}^{2} \\
& +\langle Z, U \circ Y-N\rangle \\
& +\frac{\lambda_{1}}{2}\|U \circ Y-N\|_{E}^{2} \\
& +\delta g(Y)+\frac{\lambda_{2}}{2} g^{2}(Y) .
\end{aligned}
$$

In the ALM calculation, the most significant advance is limiting $V$ by keeping $Z, \delta, \lambda_{1}$, and $\lambda_{2}$ fixed:

$\min _{Y} V\left(Y, Z, \delta, \lambda_{1}, \lambda_{2}\right)$.

This is an easy arched advancement issue, which can likewise be tackled by inclination based technique. The subordinate of $V\left(Y, Z, \delta, \lambda_{1}, \lambda_{2}\right)$ concerning $Y$ is

$$
\begin{aligned}
& \frac{\partial V\left(Y, Z, \delta, \lambda_{1}, \lambda_{2}\right)}{\partial Y}= \frac{\partial R t\left((L(Y))^{\frac{1}{2}}\right)}{\partial Y}+\beta Y P P^{R} \\
&+U \circ Z+\lambda_{1} U \circ(U \circ Y-N) \\
&+\delta \frac{\partial g(Y)}{\partial Y}+\lambda_{2} \frac{\partial g(Y)}{\partial Y} g(Y) . \\
& \text { The term } \frac{\partial R t\left((L(Y))^{\frac{1}{2}}\right)}{\partial Y} \text { can be inferred as }
\end{aligned}
$$

$$
\begin{aligned}
\frac{\partial R t\left((L(Y))^{\frac{1}{2}}\right)}{\partial Y_{j i}} & =\frac{\partial e(H)}{\partial Y_{j i}}=\sum_{l}^{M} \sum_{k}^{M} \frac{\partial e(H)}{\partial H_{l k}} \frac{\partial H_{l k}}{\partial Y_{j i}} \\
& =\operatorname{Rt}\left(\left(\frac{\partial e(H)}{\partial H}\right)^{R} \frac{\partial H}{\partial Y_{j i}}\right),
\end{aligned}
$$

where $Y_{j i}$ is the component in the $j$ th push and $i$ th segment of $Y, H_{l k}$ means the component in the $k$ th push and $l$ th segment of $H, e(H)=R t\left(H^{\frac{1}{2}}\right), H=L(Y)$. At that point

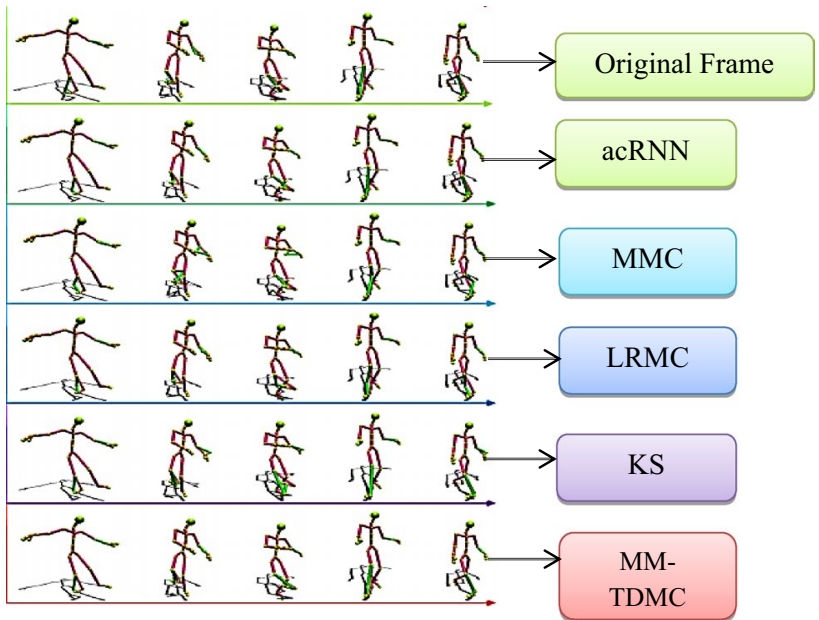

Fig. 3 Database has taken for analysis [20]

$\frac{\partial e(H)}{\partial H}=\frac{1}{2} H^{\frac{1}{2}}=\frac{1}{2}(L(Y))^{\frac{1}{2}}$,

where $\frac{\partial H}{\partial Y_{j i}}$ is not advantageous to express, so we state of $\frac{\partial l_{B}\left(y_{l}, y_{k}\right)}{\partial y_{j}}$ in the valuable material, where $H_{l k}=H_{l k}=l_{B}\left(y_{l}, y_{k}\right)$, and $y_{j}=\left[\begin{array}{c}Y_{j 1} \\ Y_{j 2} \\ \vdots \\ Y_{j(3 e)}\end{array}\right]$.

The researchers can utilize APG to limit $V\left(Y, Z, \delta, \lambda_{1}, \lambda_{2}\right)$ with $Z, \delta, \lambda_{1}$, and $\lambda_{2}$ fixed. The improvement procedure is like Algorithm 1, so the researchers don't demonstrate the subtleties in the study yet put them in the valuable data. Here, the researchers give Algorithm 2 to unravel the complete target work (21).

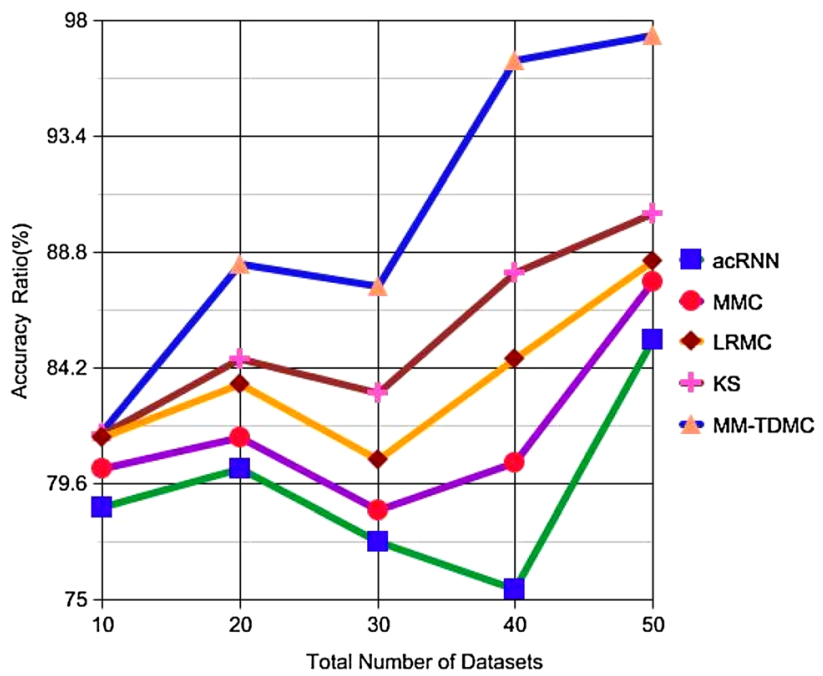

Fig. 4 Accuracy of MM-TDMC method 
Algorithm 2: optimization of equation (19) using learning parameters.

Input :

$$
\begin{gathered}
N, U, P, \beta, e, M, b>1, \quad \text { Initialize } \quad X^{(0)}=N / \max \left(\|N\|_{2},\|N\|_{\infty}\right), \quad \text { and } \quad \text { let } \\
\theta^{(0)}=1, j=0, \delta^{(0)}=1, \lambda_{1}^{(0)}=5, \lambda_{2}^{(0)}=5, j=0
\end{gathered}
$$

$1:$ Repeat

2:solve the problem of $\min _{Y} V\left(Y, Z^{(j)}, \delta^{(j)}, \lambda_{1}^{(j)}, \lambda_{2}^{(j)}\right)$ with initialization of $\mathrm{Y}^{(\mathrm{i})}$ and obtain $Y^{(j+1)}$

$3: Z^{(j+1)}=Z^{(j)}+\lambda_{1}^{(j)}\left(U \circ Y^{(j+1)}-N\right)$

4: $\delta^{(j+1)}=\delta^{(j)}+\lambda_{2}^{(j)} g\left(Y^{(j+1)}\right)$

$5: \lambda_{1}^{(j+1)}=b \lambda_{1}^{(j)}$

6: $\lambda_{2}^{(j+1)}=b \lambda_{2}^{(j)}$

7: $j=j+1$

8:until $L(C))^{\frac{q}{2}}$ convergence or reach the maximum iteration times.

Output : $Y^{(j)}()$

When the researchers get the ideal $Y$, the recuperation is done, and $Y$ is only the recouped movement grid. Like kernelized LRR. The researchers likewise utilize a part stunt, yet the utilization of Schatten $p$-standard and inclination based improvement calculation empower to deal with the movement information in its posture capacity legitimately, so the researchers achieve nonlinear information recuperation function and dodge the pre-picture issue at the same time.

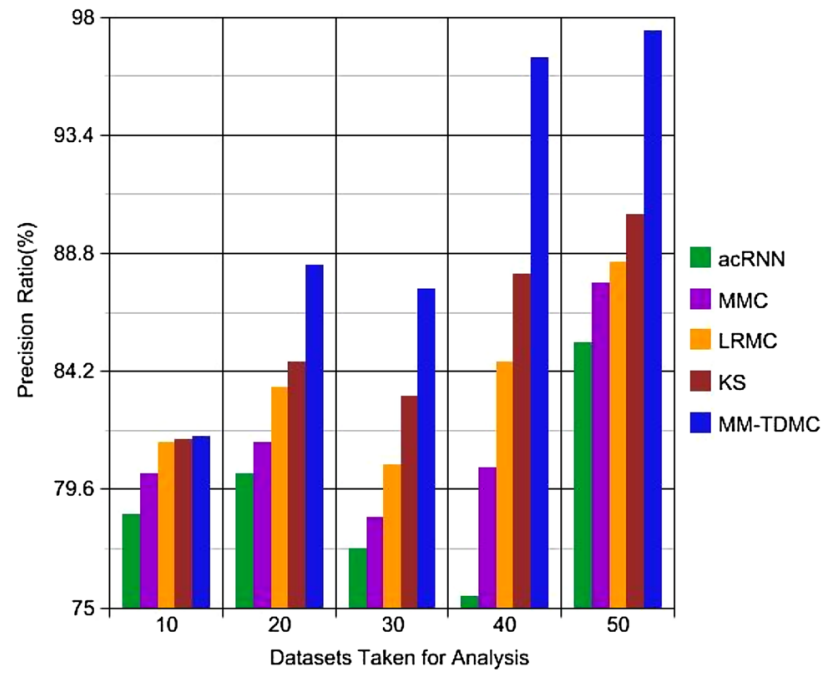

Fig. 5 Precision rate factor of MM-TDMC method

\section{Experimental results and discussion}

From the database [20], as shown in Fig. 3 in the structure of human catches the development of human beings is onechart-structured, and each side of each joint is recorded as places in a skeleton. However, positions of particular joints may be lost due to the barrier created by the body [21] or dress of the on-screen character, as is the progression of the halfway growth. To overcome this problem, MM-TDMC is used.

It is given a better accuracy rate when compared to acRNN, MMC, LRMC, and KS methods. Figure 4 shows the accuracy of the MM-TDMC process.

1. The accuracy of a measurement is how close a result comes to the actual value:

\%error $=\frac{\text { error }}{\text { actual value }} \times 100$.

2. Accuracy is the ability of the instrument to measure the accurate value. In other words, it is the closest to the normal or the actual cost of the measured value.

3. The accuracy of the total amount of instances analyzed for various motion as described in terms of the proportion of real outcomes (both true positives and true negatives).

As proposed method having a huge $\mu$ and $\nu$, the development issue (12) is simple and arched around $\operatorname{diag}\left(B B^{R}\right)=1$, which can be effectively unraveled by various inclination based development calculations, for example, the quickened proximal angle technique (APG). The subsidiary of $Q(B, C$, $\mu, \nu$ ) concerning $B$, The accuracy has been improved in our proposed method, which has been shown in Fig. 4: 
Table 1 Error rate numerical ratio

\begin{tabular}{llllll}
\hline $\begin{array}{l}\text { Error rate } \\
\begin{array}{l}\text { Number of } \\
\text { nodes }\end{array}\end{array}$ & acRNN & MMC & LRMC & KS & MM-TDMC \\
\hline 10 & 0.97 & 0.77 & 0.75 & 0.62 & 0.57 \\
20 & 0.99 & 0.79 & 0.65 & 0.65 & 0.59 \\
30 & 0.91 & 0.81 & 0.73 & 0.69 & 0.62 \\
40 & 0.93 & 0.83 & 0.80 & 0.71 & 0.64 \\
50 & 0.95 & 0.85 & 0.75 & 0.73 & 0.66 \\
\hline
\end{tabular}

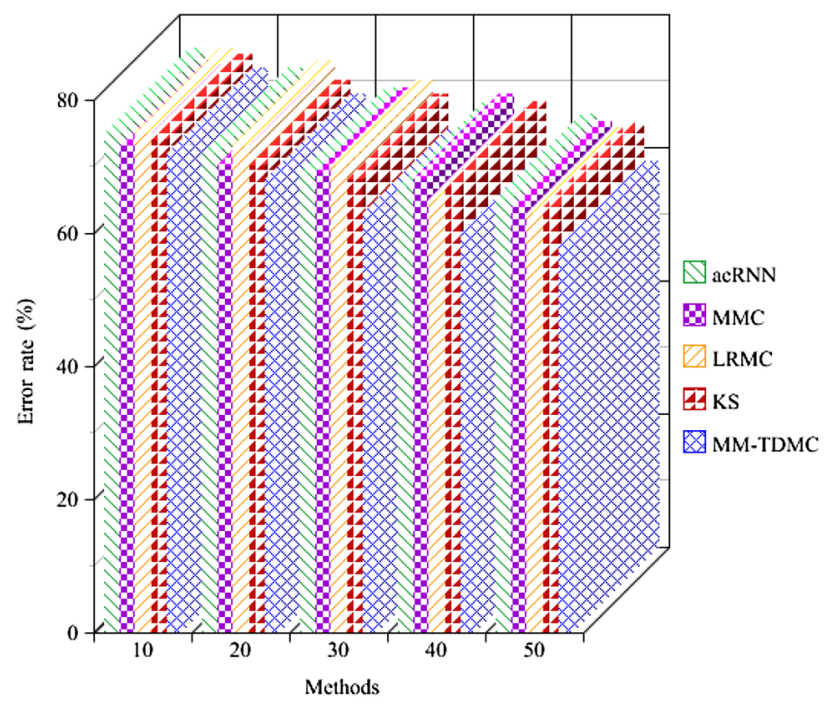

Fig. 6 Error rate analysis

\section{Binary classification}

Accuracy $=\frac{(\mathrm{TP}+\mathrm{TN})}{(\mathrm{TP}+\mathrm{TN}+\mathrm{FP}+\mathrm{FN})}$,

where TP is the true positive; FP is the false positive, TN is the true negative, FN is the false negative. Where, TP is the true positive.

One of the most efficient methods of digitizing human motions and applications in computer graphics is the motion capture system. However, the system only gives low-level data. Motion graph can generate more variable movements using signal processing methods or with space-times requirements. To overcome this problem, MM-TDMC is used, which shows a higher precision ratio. Figure 5 shows the precision rate of MM-TDMC method.

1. The term precision means two or more values of the measurements are close to each other.

2. Accuracy refers to how well measurements agree with each other in multiple tests on dance movements.

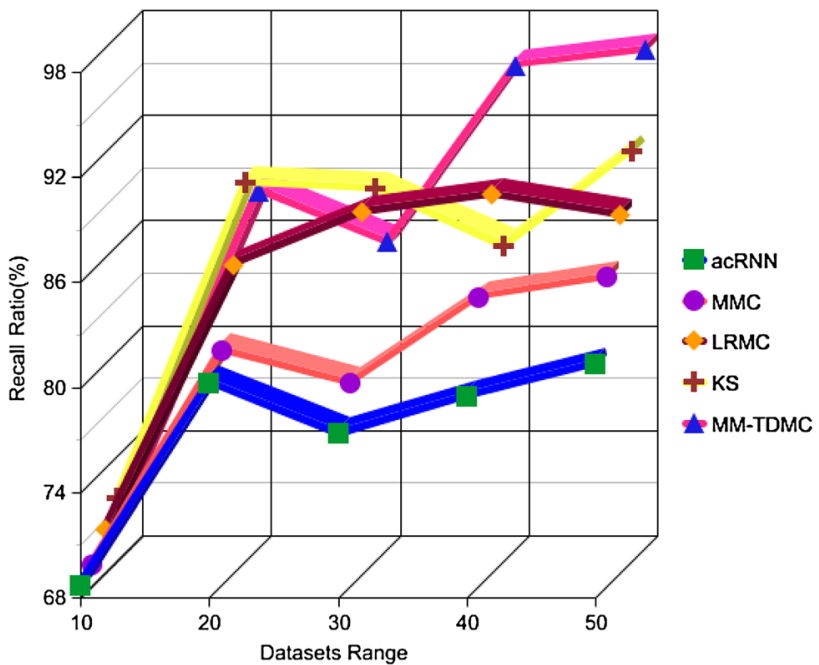

Fig. 7 Recall rate of MM-TDMC method

Table 2 Performance ratio numerical study

\begin{tabular}{llllll}
\hline $\begin{array}{l}\text { Number of } \\
\text { nodes }\end{array}$ & acRNN & MMC & LRMC & KS & MM-TDMC \\
\hline 10 & 63.5 & 68.5 & 70.42 & 73.87 & 73.5 \\
20 & 74.4 & 79.9 & 81.79 & 84.88 & 94.4 \\
30 & 75.2 & 80.3 & 82.77 & 85.00 & 95.2 \\
40 & 76.5 & 81.2 & 83.65 & 86.77 & 95.5 \\
50 & 91.8 & 82.5 & 84.77 & 87.38 & 96.8 \\
\hline
\end{tabular}

3. Precision is how consistent results are when measurements are repeated:

Precision $=\frac{\mathrm{TP}}{\mathrm{TP}+\mathrm{FP}}$.

where TP is the true positive; FP is the false positive.

Because of capture systems constraints, the raw information collected invariably contain missing information. All current techniques use the convex nuclear standards to overcome the resulting issue of ranking minimization known as NP-hard as a ranking replacing the convexity of the objective function. This paper which has high Error recovery and human intuition agreement. MM-TDMC which have a minimum error rate when compared to acRNN, MMC, LRMC, and KS methods. Figure 6 shows the error rate of the MMTDMC process.

1. Error rates refer to the frequency of errors that occurred, defined as the ratio of the full amount of error units to the number of transmitted data units for dance analysis, as shown in Table 1: 


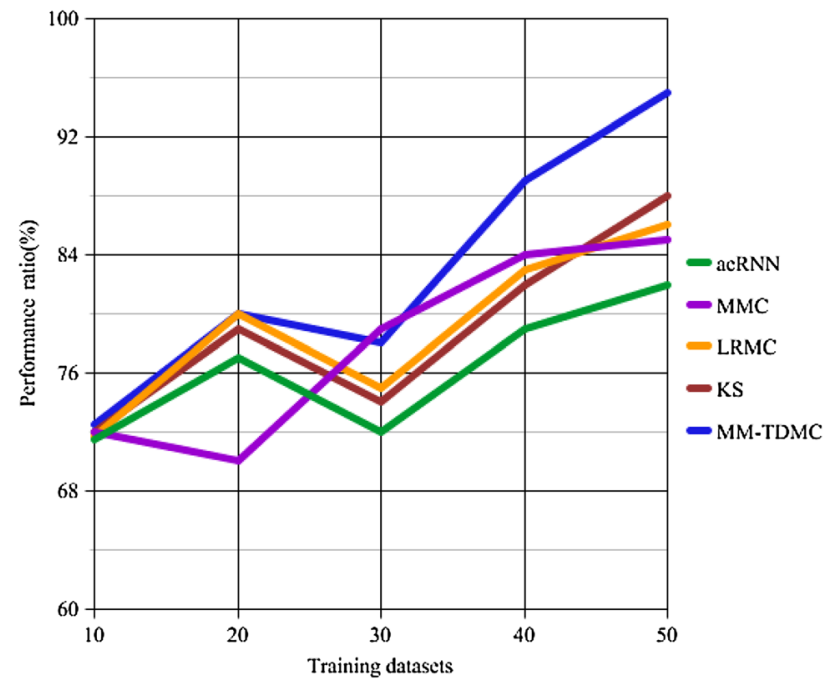

Fig. 8 Performance ratio of MM-TDMC method

$$
\text { Error rate }=\frac{\text { Total number of data units resulted in error }}{\text { Total number of unites data transmitted }} .
$$

2. It measures a communication channel's efficiency. It is the relation between the amount of incorrect information about the dance movements and the entire amount of information units transferred.

The machine learning-based two-dimensional matrix computation model (MM-TDMC) which have a better recall rate when compared to acRNN, MMC, LRMC, and KS methods. Figure 7 shows the recall rate of the MM-TDMC process. The recall is the percentage of positive results correctly predicted in the actual class of all observations:

Recall $=\mathrm{TP} / \mathrm{TP}+\mathrm{FN}$,

where TP is the true positive; FN is the false negative.

A wide range of applications has distinct methods for representing human movements and recognition. Analysis of human motion is the most general word, and implementation determines the amount and length of movement of the body components. MM-TDMC achieves higher performance when compared to acRNN, MMC, LRMC, and KS methods, as shown in Table 2.

Figure 8 shows the performance ratio of the MM-TDMC method, as stated in the proposed way MM-TDMC, Where $L_{\sigma n}\left(C_{i}\right)$ is the piece framework of the $i$ th joint characterized on the part $l_{\sigma n}(\cdot, \cdot)$. When executing the learning procedure, let $q=1 / 16$ for the Schatten $p$-standard to make an adequately precise estimate of rank $(C)$. As indicated by APG, the researchers give Algorithm 1 to take care of the issue of Eq. (13). At that point, the researchers use a PH calculation to illuminate the entire numerous portion learning issue (11). Since PH calculation is anything but difficult to execute, the researchers do not demonstrate the calculation subtleties here yet adjacent it in the strengthening data, and the performance ratio has been discussed in Fig. 8 .

Here, the experimental validation shows that propose MM-TDMC for human motion and dance recovery shows extensive experimental results and comparisons with acRNN, MMC, LRMC, and KS methods.

\section{Conclusion}

In this paper, the researchers present a MM-TDMC for human motion and dance recovery. In the suggested model, the theoretical guarantee for recovering data from nonlinear motion is missing in the two-dimensional matrix computer model for linear data instead of using a current method. The experimental results and discussion section shows the MMTDMC, which gives promising outcomes when compared to other methods (acRNN, MMC, LRMC, KS).

Open Access This article is licensed under a Creative Commons Attribution 4.0 International License, which permits use, sharing, adaptation, distribution and reproduction in any medium or format, as long as you give appropriate credit to the original author(s) and the source, provide a link to the Creative Commons licence, and indicate if changes were made. The images or other third party material in this article are included in the article's Creative Commons licence, unless indicated otherwise in a credit line to the material. If material is not included in the article's Creative Commons licence and your intended use is not permitted by statutory regulation or exceeds the permitted use, you will need to obtain permission directly from the copyright holder. To view a copy of this licence, visit http://creativecommons.org/licenses/by/4.0/.

\section{References}

1. Xia G, Sun H, Zhang G, Feng L (2016) Human motion recovery jointly utilizing statistical and kinematic information. Inf Sci 339:189-205

2. Peng XB, Abbeel P, Levine S, van de Panne M (2018) Deepmimic: Example-guided deep reinforcement learning of physicsbased character skills. ACM Trans Graphics (TOG) 37(4):143

3. Wyon MA, Twitchett E, Angioi M, Clarke F, Metsios G, Koutedakis Y (2011) Time motion and video analysis of classical ballet and contemporary dance performance. Int J Sports Med 32(11):851-855

4. Suzuki K, Yataka K, Okumiya Y, Sakakibara S, Sako K, Mimura H, Inoue Y (2016) Rapid-response, widely stretchable sensor of aligned MWCNT/elastomer composites for human motion detection. ACS Sens 1(6):817-825

5. Aristidou A, Cohen-Or D, Hodgins JK, Shamir A (2018) Selfsimilarity analysis for motion capture cleaning. In: Computer graphics forum, vol 37, no 2, pp 297-309

6. Argall BD, Billard AG (2010) A survey of tactile human-robot interactions. Robot Auton Syst 58(10):1159-1176

7. Peng SJ, He GF, Liu X, Wang HZ (2015) Hierarchical block-based incomplete human mocap data recovery using adaptive nonnegative matrix factorization. Comput Graphics 49:10-23 
8. Lee D, Nakamura Y (2014) Motion recognition and recovery from occluded monocular observations. Robot Auton Syst 62(6):818-832

9. Peng XB, Kanazawa A, Malik J, Abbeel P, Levine S (2018) Sfv: reinforcement learning of physical skills from videos. In: SIGGRAPH Asia 2018 technical papers. ACM, p 178

10. Wei K, Kording KP (2018) Behavioral tracking gets real. Nat Neurosci 21(9): 1146

11. Xia G, Sun H, Chen B, Liu Q, Feng L, Zhang G, Hang R (2018) Nonlinear low-rank matrix completion for human motion recovery. IEEE Trans Image Process 27(6):3011-3024

12. Li Z, Zhou Y, Xiao S, He C, Huang Z, Li H (2017) Auto-conditioned recurrent networks for extended complex human motion synthesis. arXiv: 1707.05363

13. Essid S, Lin X, Gowing M, Kordelas G, Aksay A, Kelly P, Tournemenne R (2013) A multi-modal dance corpus for research into interaction between humans in virtual environments. J Multimodal User Interfaces 7(1-2): 157-170

14. Shakeel PM, Baskar S, Dhulipala VS, Mishra S, Jaber MM (2018) Maintaining security and privacy in health care system using learning based deep-Q-networks. J Med Syst 42(10):186

15. Kitsikidis A, Dimitropoulos K, Douka S, Grammalidis N (2014) Dance analysis using multiple kinect sensors. In: 2014 international conference on computer vision theory and applications (VISAPP), vol 2. IEEE, pp 789-795

16. Li C, Chen H, Xue D, Hu Z, Zhang L, He L, Sun H (2019) Weakly supervised cervical histopathological image classification using multilayer hidden conditional random fields. In: International conference on information technologies in biomedicine. Springer, Cham, pp 209-221

17. Baskar S, Dhulipala VS, Shakeel PM, Sridhar KP, Kumar R (2019) Hybrid fuzzy based spearman rank correlation for cranial nerve palsy detection in MIoT environment. Health Technol. https ://doi.org/10.1007/s12553-019-00294-8

18. Yun S, Choi J, Won CS (2019) Omnidirectional 3D point clouds using dual kinect sensors. J Sens 2019:6295956

19. Ma Z, Wu J, Zhong SH, Heinen SJ (2019) Object-based and multiframe motion information predict human eye movement patterns during video viewing. Electron Imaging 2019(12):205-211

20. Yu T, Zheng Z, Guo K, Zhao J, Dai Q, Li H, Liu Y (2018) Doublefusion: real-time capture of human performances with inner body shapes from a single depth sensor. In: Proceedings of the IEEE conference on computer vision and pattern recognition, pp 7287-7296

21. Manogaran G, Shakeel PM, Fouad H, Nam Y, Baskar S, Chilamkurti N, Sundarasekar R (2019) Wearable IoT smart-log patch: an edge computing-based Bayesian deep learning network system for multi access physical monitoring system. Sensors 19(13):3030

Publisher's Note Springer Nature remains neutral with regard to jurisdictional claims in published maps and institutional affiliations. 